\title{
Congenital cystic adenomatoid malformation of the lung presenting as pyopneumothorax in an eighteen-year-old woman
}

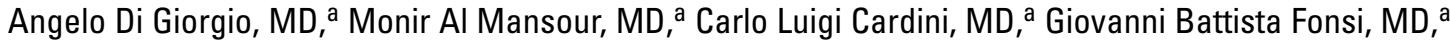 \\ Giuseppe Carmenini, MD, and Nicola Gueli, MD, Rome, Italy
}

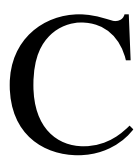

ongenital cystic adenomatoid malformation of the lung (CCAM) is an uncommon developmental abnormality usually presenting as respiratory distress in the neonatal period. Presentation in adulthood is rare and few cases have been reported in the literature. We report a case of CCAM presenting as pyopneumothorax in an 18-year-old woman.

\section{Clinical Summary}

An 18-year-old woman was admitted to our department with a sudden onset of high fever, shortness of breath, right-sided chest pain, and productive cough. The patient had a history of frequent recurrent episodes of pneumonia starting at the age of 5 years, for which she had been treated with antibiotics and drugs to relieve symptoms. Other clinical signs were absent. Laboratory parameters showed a significant increase in white blood cells $\left(>19,500 \mathrm{~mm}^{3}\right)$. The initial chest $\mathrm{x}$-ray film showed a sizable infiltration in the right superior pulmonary fields with ipsilateral pneumothorax and pleural effusion. A computed tomographic scan of the chest showed a single large broken cyst confined to the right upper lobe with evidence of ipsilateral pneumothorax, pleural effusion, and contralateral mediastinal shift (Figure 1). Static and dynamic ventilatory function parameters demonstrated an abnormal obstructive pattern: forced expiratory volume in 1 second was 1.60 (34\%), forced vital capacity was $1.25(36 \%)$, and total lung capacity was 2.24 (50\%); residual volume was not significantly affected. Ventilatory scintigraphy did not reveal ventilated parenchyma in the right lung, but the left lung was totally ventilated. Bronchoscopic findings showed no abnormalities, and cardiac function was within normal limits.

Initially, a chest tube was inserted to allow right lung reexpansion and pleural drainage of $500 \mathrm{~mL}$ of purulent fluid. Ten days later the patient underwent open surgical exploration through a posterolateral thoracotomy in the fifth intercostal space. Pleural

\footnotetext{
From the Departments of Surgery, "Pietro Valdoni," and Internal Medicine, ${ }^{\text {b }}$ University of Rome "La Sapienza," Rome, Italy.

Received for publication March 1, 2001; accepted for publication March 27, 2001.

Address for reprints: Angelo Di Giorgio, MD, Università degli Studi di Roma "La Sapienza," Dipartimento di Chirurgia "Pietro Valdoni" Policlinico Umberto I, Via Lancisi 2, 00161, Roma, Italia (E-mail: angelo.digiorgio@uniroma1.it).

J Thorac Cardiovasc Surg 2001;122:1034-6

Copyright $\odot 2001$ by The American Association for Thoracic Surgery

$0022-5223 / 2001 \$ 35.00+0 \quad \mathbf{1 2 / 5 4 / 1 1 6 1 9 3}$

doi:10.1067/mtc.2001.116193
}

thickening and strong pleural adhesions were present throughout the pleural cavity, which appeared full of purulent fluid and mucoid material. The superior lobe was completely affected, with a central large necrotic gangrenous area containing purulent fluid and a web of denuded vascular and bronchiolar structures. After lavage of the pleural cavity and complete lysis of adhesions, the middle and inferior lobes, previously totally collapsed, appeared anatomically and functionally preserved. Right upper lobectomy was performed with biopsy of the parietal pleura and excision of some lobar and interlobar nodes.

Macroscopic examination of the resected lobe showed a large multiloculated cystic area with a centrally located, broken cavity about $15 \mathrm{~cm}$ in diameter. The area was surrounded by smaller cystic lesions containing mucoid material. The histologic picture showed an irregular proliferation of cystic structures resembling bronchioles intermingled with fibrous connective septa lined by tall columnar respiratory epithelium (Figure 2). The features were those of type I CCAM according to a modification of Stocker, Madewell, and Drake's classification. ${ }^{2}$ The postoperative course was uneventful and the patient was discharged on the 20th postoperative day. She is doing well 5 years after the operation with complete functional recovery.

\section{Discussion}

CCAM is a rare congenital disorder of pulmonary development first described by Chin and Tang ${ }^{1}$ in 1949. This entity is characterized by multiple solid cystic lesions that may produce respiratory distress in the near-term infant at birth, and it is sometimes associated with fetal anasarca, ascites, and maternal polyhydramnios. No sex or race predilection has been identified. The malformation is usually unilateral and sublobar or lobar in size, but occasionally it can be multilobar. Stocker, Madewell, and Drake ${ }^{2}$ classified these lesions on the basis of the clinical presentation and pathologic picture. Traditionally, CCAM can be classified into 3 different patterns on the basis of the size of the cysts and histologic findings: Type $I$ is composed of single or multiple large cysts $(>2 \mathrm{~cm}$ in diameter) lined with ciliated columnar epithelium and mucus secreting cells; type II is composed of multiple small cysts $(<1 \mathrm{~cm})$ lined with ciliated cuboidal to columnar epithelium without mucous secreting cells; type III is a large bulky mass made up of tiny cysts $(<0.5 \mathrm{~cm})$ lined with cuboidal epithelium. Type I accounts for $70 \%$ of CCAMs, type II $20 \%$, and type III $10 \%$. Moreover, type II is most frequently associated with other congenital anomalies, such as extralobar sequestration, diaphragmatic hernia, and renal agenesis or dysgenesis. ${ }^{2}$

Presentation during adulthood is rare and has usually been that of recurrent pulmonary infection, most often involving a 

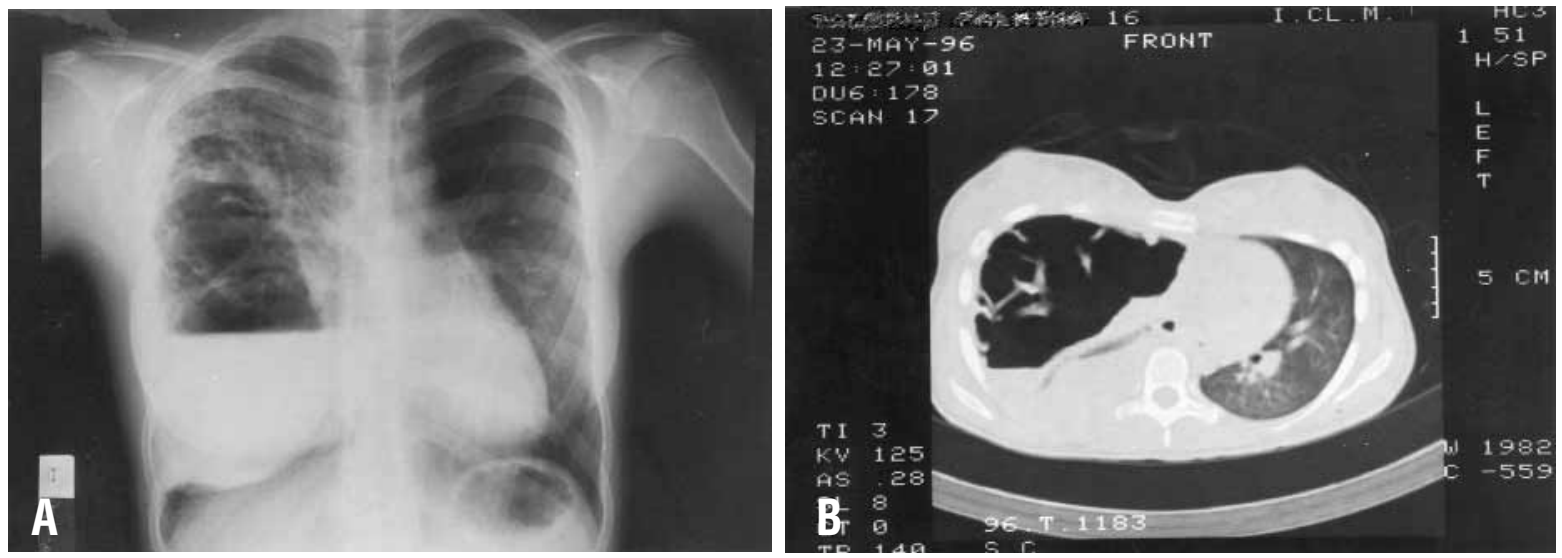

Figure 1. A, Preoperative $x$-ray film of the chest showing the lesion appearing as a sizable infiltration in the right lung with pneumothorax and pleural effusion. B, Preoperative computed tomographic scan showing a large broken cyst in the right lung with evidence of ipsilateral pneumothorax, pleural effusion, and contralateral mediastinal shift.
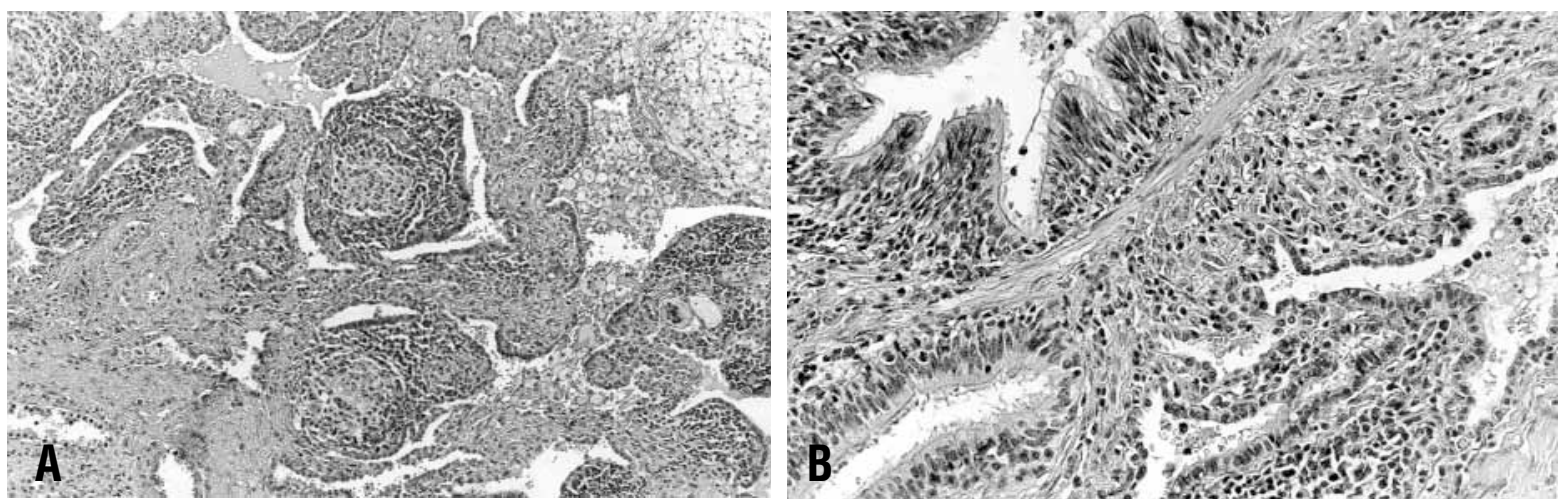

Figure 2. A, Irregular proliferation of cystic structures resembling bronchioles intermingled with fibrous connective septa with signs of inflammatory reaction in the surrounding lung parenchyma (hematoxylin and eosin; original magnification $\times 100)$. B, Microscopic cysts lined by tall columnar epithelium (hematoxylin and eosin; original magnification $\times 400)$.

single lobe. Chest radiographic findings are variable and depend on the type of lesion and infectious complications; often a depression of the ipsilateral diaphragm and contralateral mediastinal shift are visible. Computed tomographic scans of the thorax are helpful to diagnose the nature of the cyst. A differential diagnosis is necessary to distinguish CCAM from other rare cystic tumors. Up to the year 2000, only 24 cases of CCAM in adulthood have been reported in the literature, 12 in men and 12 in women. The age distribution among the reported cases has ranged from 18 to 65 years with a mean age of 30.3 years. In 8 cases CCAM was asymptomatic and was diagnosed from routine $\mathrm{x}$-ray films of the chest. Nine cases involved a history of unresolving or recurrent pulmonary infection. Three cases were revealed by spontaneous pneumothorax, 3 by hemoptysis, and 1 by dyspnea. Seventeen of these cases corresponded to type I
CCAM. The malformation was limited to one lower lobe in 16 patients, one upper lobe in 2 , one middle lobe in 1 , and a lower and middle lobe in 1 case. Bilateral lung involvement was described in 1 case. In 3 cases side and site were not identified. In 4 cases of type I CCAM the primary lesion was associated with bronchoalveolar carcinoma. ${ }^{3,4}$

Management of CCAM of the lung is no longer controversial. Most authors recommend surgical resection to confirm the diagnosis and lessen the risk of infection or malignant degeneration; moreover, the prognosis after radical excision seems to be excellent. ${ }^{5,6}$ According to this analysis, we conclude that prognosis and survival in the older child or adult depend on histologic type, infectious complications, and malignant potential of these lesions. Therefore, surgical exploration and sequential excision are mandatory in all cases, even in patients who are free of symptoms. 


\section{References}

1. Chin KY, Tang MY. Congenital adenomatoid malformation of one lobe of a lung with general anasarca. Arch Pathol Lab Med. 1949;48:221-9.

2. Stocker JT, Madewell JE, Drake RM. Congenital cystic adenomatoid malformation of the lung. Hum Pathol. 1977;8:155-71.

3. Hellmuth D, Glerant JC, Sevestre H, Remond A, Jounieaux V. Pulmonary adenomatoid malformation presenting as unilobar cyst in an adult. Respir Med. 1998;92:1364-7.
4. Dahabreh J, Zisis C, Vassiliou M, Arnogiannaki N. Congenital cystic adenomatoid malformation in an adult presenting as lung abscess. Eur J Cardiothorac Surg. 2000;18:720-3.

5. Case records of the Massachussets General Hospital (case 20-1996). $N$ Engl J Med. 1996;334:1726-32.

6. Evrard V, Ceulemans J, Coosemans W, De Baere T, De Leyn P, Deneffe G, et al. Congenital parenchymatous malformations of the lung. World J Surg. 1999;23:1123-32. 